Check for updates

Cite this: RSC Adv., 2017, 7, 21618

Received 23rd February 2017

Accepted 5th April 2017

DOI: $10.1039 / \mathrm{c} 7 \mathrm{ra02267k}$

rsc.li/rsc-advances

\section{Facile synthesis of AgNPs on reduced graphene oxide for highly sensitive simultaneous detection of heavy metal ions}

\author{
Shengbo Sang, ${ }^{a}$ Dan Li, ${ }^{a}$ Hui Zhang, ${ }^{\text {bc }}$ Youyi Sun, ${ }^{\text {bc }}$ Aoqun Jian, ${ }^{a}$ Qiang Zhang ${ }^{a}$ \\ and Wendong Zhang (iD *b
}

Silver nanoparticles grown on reduced graphene oxide (AgNPs/RGO) were successfully synthesized via a facile in situ method. The samples were characterized by scanning electron microscopy (SEM), Raman spectroscopy, field emission transmission electron microscopy (FETEM) and X-ray diffraction (XRD). The results show that silver ions distribute extensively on the RGO sheets. In order to prepare an electrochemical sensor, the AgNPs/RGO nanocomposite was used to modify the surface of a magnetic glassy carbon electrode. The device was characterized by cyclic voltammetry (CV) and electrochemical impedance spectroscopy (EIS). The as-prepared AgNPs/RGO nanocomposite was measured with square wave anodic stripping voltammetry (SWASV), and exhibited excellent electrochemical activity and sensitivity towards the detection of heavy metal ions, including $\mathrm{Pb}(॥), \mathrm{Cd}\left(\|_{1}\right) \mathrm{Cu}(॥)$ and $\mathrm{Hg}(॥)$. The sensitivities were respectively $48.69,40.06,15.66$ and $43.18 \mu \mathrm{A} \mu \mathrm{M}^{-1}$ and the limits of detection (LOD) were respectively $0.141,0.254,0.178$ and $0.285 \mu \mathrm{M}$. Compared to the bare RGO film, the AgNPs/RGO nanocomposite showed a significantly higher activity for anodic stripping analysis of the four considered heavy metal ions; in particular, the peak current enhancement was about 1.5 times higher for $\mathrm{Pb}(I)$. Experiments also show that the AgNPs/RGO-modified electrode displays good anti-interference properties. Moreover, this study provides a potential material for electrochemical detection of heavy metal ions, individually or simultaneously.

\section{Introduction}

It is well documented that heavy metal pollutants, including $\mathrm{Cd}(\mathrm{II}), \mathrm{Pb}$ (II), $\mathrm{Cu}$ (II) and $\mathrm{Hg}$ (II) ions, have attracted a great deal of attention due to their high toxicity, easy accumulation and lack of degradability. This type of pollutant could be seriously hazardous not only for the environment around biological systems but also directly for human beings. ${ }^{1-6}$ Therefore, it is significant to develop direct and highly sensitive tools to determine the concentration of heavy metals in solutions. To date, many kinds of techniques have been applied for the detection of heavy metal ions, including mass spectrometry, ${ }^{7}$ optical methods ${ }^{8}$ and electrochemical methods. ${ }^{9}$ Recent developments in materials science and technology have enabled rapid electrochemical analysis with low power consumption, high sensitivity and wide adaptability, ${ }^{10}$ and therefore

\footnotetext{
${ }^{a}$ MicroNano System Research Center, Key Lab of Advanced Transducers and Intelligent Control System of the Ministry of Education, College of Information Engineering, Taiyuan University of Technology, Taiyuan 030024, China

${ }^{b}$ School of Materials Science and Engineering, North University of China, Taiyuan 030051, P. R. China.E-mail: mnsrc_tyut@163.com; syyi2010@163.com

${ }^{c}$ Shanxi Province Key Laboratory of Functional Nanocomposites, North University of China, Taiyuan 030051, P. R. China
}

electrochemical analysis methods for detecting heavy metals certainly have great potential. A key step for electrochemical analysis methods to detect heavy metal ions is the design and synthesis of the working electrode materials. Among the working electrode materials, it is difficult to ignore metal nanoparticlemodified electrodes, which have been proven to display remarkable electrochemical sensitivity thanks to their large specific surface area, fast electron transfer and high density of active sites. ${ }^{\mathbf{1 1} 12}$ Among the different metal nanoparticles, silver nanoparticles (AgNPs) show interesting quantum characteristics, allowing for a large specific surface area, small particle diameter, fast electron transfer ability, etc. Therefore, AgNPs are one of the most suitable materials to develop nanocomposite electrochemical sensors. ${ }^{13}$ So far, various nanomaterials, including noble metal nanoparticles (NPs), carbon nanotubes (CNTs) and graphene, have been applied to fabricate electrochemical sensors. ${ }^{14-17}$ In addition, graphene oxide and reduced graphene oxide (RGO) have attracted great attention thanks to their high sensitivity, low cost and eco-friendly properties. In the past few years, a series of carbonaceous materials, including graphene, ${ }^{\mathbf{1 8 , 1 9}}$ carbon nanotubes (CNTs), ${ }^{\mathbf{2 0 - 2 2}}$ carbon paste, carbon nanospheres $^{23}$ and porous carbons, ${ }^{24,25}$ have been used to make efficient electrochemical sensors. Several groups have investigated graphene oxide and metal nanoparticle composite electrodes for 
electrochemical sensors. Periyasami Gnanaprakasam et al. have fabricated AuNPs on reduced graphene oxide for the simultaneous detection of toxic heavy metal ions and bioremediation using bacteria as the scavenger. ${ }^{26}$ Riyaz Ahmad Dar et al. have prepared an AgNPs/GO composite and applied it for As(III) detection. ${ }^{27}$ Yan Wei et al. have fabricated a $\mathrm{SnO}_{2} / \mathrm{RGO}$ nanocomposite for the simultaneous electrochemical detection of heavy metal ions.$^{28}$ However, so far, AgNPs/RGO nanocomposites have rarely been reported to be applicable for the detection of other kinds of heavy metal ions, including $\mathrm{Pb}$ (II), $\mathrm{Cd}(\mathrm{II}), \mathrm{Cu}$ (II) and $\mathrm{Hg}$ (II). Hence, it is meaningful to investigate the use of AgNPs/ RGO nanocomposites as an electrochemical sensing material to individually and simultaneously detect heavy metal ions through square wave anodic stripping voltammetry (SWASV).

Based on the above considerations, in this work we applied a facile approach to synthesize RGO and different mass ratio AgNPs/RGO nanocomposites, which were applied as electrochemical sensors to detect heavy metal ions. Specifically, the AgNPs/RGO nanocomposite was deposited on the surface of a magnetic glassy carbon electrode to act as a novel electrochemical sensor for detecting and quantifying environmentally toxic elements, including $\mathrm{Pb}$ (II), $\mathrm{Cd}$ (II), $\mathrm{Cu}$ (II) and $\mathrm{Hg}$ (II). The sensor could not only adsorb all kinds of heavy mental ions due to its large electrochemically active surface area, but it also ensured a fast and high sensitivity current response thanks to the high electron transfer speed between the electrode and solution. Furthermore, the mutual interference and cycling stability of the working electrode were studied in detail.

\section{Experimental}

\subsection{Materials}

Analytical grade reagents were used for the synthesis of the AgNPs/RGO nanocomposites. Graphite powder (320 mesh, spectrum pure), ascorbic acid, $\mathrm{H}_{2} \mathrm{SO}_{4}, \mathrm{KMnO}_{4}, \mathrm{AgNO}_{3}$ and $\mathrm{H}_{2} \mathrm{O}_{2}$ (30 wt\%) were purchased from Sinopharm Chemical Reagents Co., Ltd., Shanghai, China and used without further purification. Stock solutions of heavy metal ions including $\mathrm{Hg}(\mathrm{II}), \mathrm{Cd}(\mathrm{II})$, $\mathrm{Pb}$ (II) and $\mathrm{Cu}$ (II) were prepared with $\mathrm{Hg}\left(\mathrm{NO}_{3}\right)_{2}, 3 \mathrm{CdSO}_{4} \cdot 8 \mathrm{H}_{2} \mathrm{O}$, $\mathrm{Pb}\left(\mathrm{NO}_{3}\right)_{2}$ and $\mathrm{Cu}\left(\mathrm{NO}_{3}\right)_{2}$. In order to regulate the $\mathrm{pH}$ value, several drops of $\mathrm{HNO}_{3}$ were added to the $\mathrm{Hg}\left(\mathrm{NO}_{3}\right)_{2}$ and $\mathrm{Pb}\left(\mathrm{NO}_{3}\right)_{2}$ stock solutions. Acetate buffer (HAc-NaAc) solutions $(0.1 \mathrm{M})$ were prepared by mixing stock solutions of $0.1 \mathrm{M}$ HAc and NaAc, and were used as the supporting electrolyte. The water used in the experiments was obtained from an ultra-pure water manufacturing system (URT-11-10T).

\subsection{Characterizations}

The morphology of the AgNPs/RGO nanocomposites was characterized by field-emission scanning electron microscopy (FESEM, Hitachi S-4700) and field emission transmission electron microscopy (FETEM, JEOL 2100). X-ray diffraction (XRD) patterns of the AgNPs/RGO nanocomposites were acquired on a Maxima 7000S XRD (Shimadzu, Japan). Raman spectra were collected on a Jobin-Yvon LabRam HR800 Raman spectrometer. Electrochemical experiments were carried out using a ZAHNER-
PP211 electrochemical workstation. A conventional threeelectrode configuration was used. The working electrode was a bare or modified magnetic glassy carbon electrode (MGCE, 3 $\mathrm{mm}$ in diameter), and an $\mathrm{Ag} / \mathrm{AgCl}(3 \mathrm{M} \mathrm{KCl})$ electrode and a platinum electrode were used as the reference and counter electrodes, respectively. Furthermore, electrochemical impedance spectra measurements were performed in $0.1 \mathrm{M} \mathrm{KCl}$ containing $1.0 \mathrm{mM} \mathrm{K}_{3}\left[\mathrm{Fe}(\mathrm{CN})_{6}\right]$. In addition, $\mathrm{pH}$ measurements were performed using a $\mathrm{pH}$ meter.

\subsection{Synthesis of the AgNPs/RGO nanocomposites}

The AgNPs/RGO nanocomposites were prepared following an in situ synthesis method. Firstly, RGO was prepared from natural graphite via a modified Hummers' method. ${ }^{43}$ Secondly, in order to prepare nanocomposites with RGO : Ag mass ratios of $1: 0.25$ and $1: 0.75,31.25$ and $93.75 \mathrm{mg}$ of $\mathrm{AgNO}_{3}$ were respectively dispersed in $50 \mathrm{~mL}$ of RGO DMF solution $\left(2.5 \mathrm{mg} \mathrm{mL}^{-1}\right)$ by stirring at $60{ }^{\circ} \mathrm{C}$ for $2 \mathrm{~h}$. During the AgNPs/RGO nanocomposite preparation process, $30 \mathrm{~mL}$ of $2.6 \%$ ascorbic acid was added to reduce the silver ions directly onto the RGO and $0.6 \mathrm{~g}$ of beta cyclodextrin was used as a stabilizing agent. Then, the AgNPs/ RGO nanocomposites with different mass ratios were obtained by centrifugation (2000 rpm). For comparison, bare RGO was also prepared under the same conditions.

\subsection{Preparation of the AgNPs/RGO/MGCE}

In order to decrease the residue on the surface of the bare MGCE, the bare MGCE was ultrasonically cleaned in ethanol for $20 \mathrm{~min}$ and then polished carefully by using $0.3 \mu \mathrm{m}$ and $0.05 \mu \mathrm{m}$ alumina slurry. The polished MGCE was washed with $\mathrm{HNO}_{3}$ $(1: 1)$, absolute ethylene, and deionized water to reduce the amount of adsorbed substances on the surface of the electrode, and then dried at room temperature. Then, $1 \mathrm{mg}$ of AgNPs/RGO nanocomposite powder was dispersed in $2 \mathrm{~mL}$ of alcohol. To create a homogeneous suspension, $20 \mathrm{~mL}$ of the solvent mixture was ultrasonically stirred for $3 \mathrm{~h}$. Subsequently, $4 \mu \mathrm{L}$ of the homogeneous suspension was added onto the MGCE surface and dried at room temperature. Hence, the modified electrode was composed of AgNPs/RGO/MGCE. In order to facilitate comparison, an RGO/MGCE was also prepared with an RGO homogeneous suspension following the same procedure.

\subsection{Simultaneous detection of heavy metal ions}

In order to realize the individual and simultaneous detection of $\mathrm{Pb}$ (II), $\mathrm{Cd}(\mathrm{II}), \mathrm{Cu}(\mathrm{II})$ and $\mathrm{Hg}(\mathrm{II})$, electrochemical analysis measurements were carried out by using SWASV. Firstly, the AgNPs/RGO/MGCE was put into a mixed metal solution including $0.1 \mathrm{M} \mathrm{HAc}-\mathrm{NaAc}$ solution $(\mathrm{pH}=5.0)$ and heavy metal ions. Then, heavy metal ions were deposited onto the surface of the working electrode at the potential of $-1.5 \mathrm{~V} v \mathrm{~s}$. SCE for $150 \mathrm{~s}$. The deposition process was used to reduce the heavy metal ions from the mixture solutions onto the working electrode. On the contrary, stripping was performed to oxidize the reduced metals. All experiments were implemented under the following experimental conditions: the scanning potential ranged from -1.0 to $0.6 \mathrm{~V}$; the amplitude was $20 \mathrm{mV}$; the step height 
potential was $5 \mathrm{mV}$; the period was $40 \mathrm{~ms}$; the time of integration was $2 \mathrm{~ms}$; the start ramp was $100 \mathrm{mV} \mathrm{s}^{-1}$; the frequency was $25 \mathrm{~Hz}$. Furthermore, a positive potential was applied to the modified electrode for $60 \mathrm{~s}$ to remove the deposited residual heavy metal ions from the surface after each detection test.

\section{Results and discussion}

\subsection{Morphological and structural characterization of the AgNPs/RGO nanocomposites}

The morphology and structure of the as-prepared AgNPs/RGO nanocomposites were characterized by SEM and TEM and shown in Fig. 1. The SEM and TEM images show that the RGO

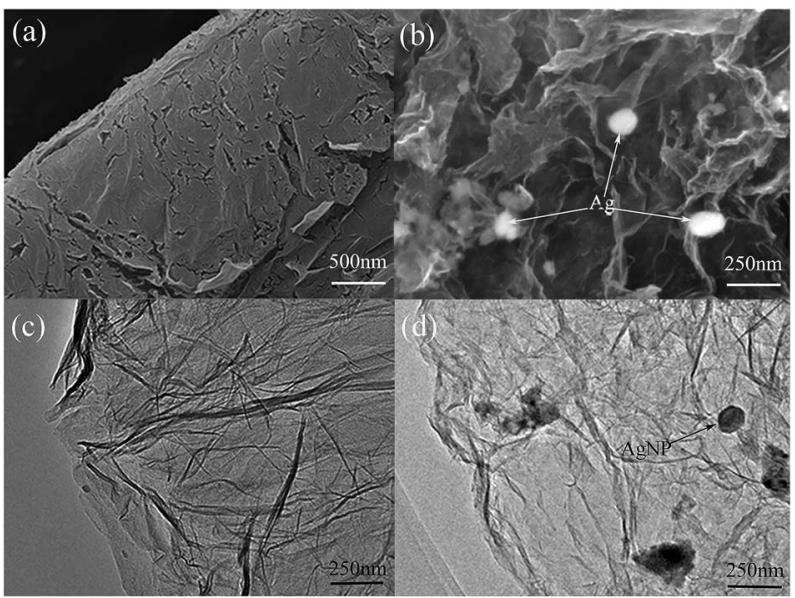

Fig. 1 SEM and TEM images of the RGO film (a and c) and the AgNPs/ RGO nanocomposite film ( $b$ and $d$ ).
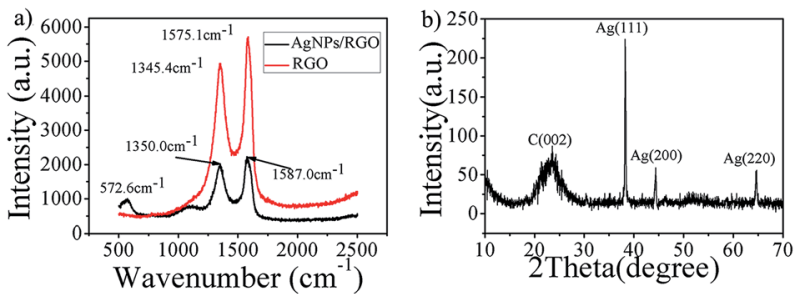

Fig. 2 (a) Raman spectra of RGO and AgNPs/RGO. (b) XRD of AgNPs/RGO. surface was crumpled and wrinkled with a multi-layer structure. Hence, it could provide a large surface area for the attachment of AgNPs. As shown in Fig. 1b, AgNPs were homogeneously distributed on the RGO sheets. This demonstrates a strong interaction between the RGO and AgNPs. Fig. 1c and d show typical TEM images of RGO and the AgNPs/RGO nanocomposites, indicating that AgNPs are attached onto the surface of the RGO nanosheets. The highly dispersed AgNPs on the RGO nanosheets probably contribute to the high electrochemical activity of the sensor.

Fig. 2a shows the Raman spectra of RGO and the AgNPs/RGO nanocomposites. Each curve displays two obvious peaks, corresponding to the $\mathrm{D}$ and $\mathrm{G}$ bands of graphene. ${ }^{36}$ The $\mathrm{D}$ and $\mathrm{G}$ bands of RGO in the AgNPs/RGO nanocomposites were respectively observed at 1350 and $1587 \mathrm{~cm}^{-1}$, while the D and G band of RGO were respectively observed at 1345 and $1350 \mathrm{~cm}^{-1}$. In addition, we calculated the $\mathrm{D} / \mathrm{G}$ intensity ratio for the $\mathrm{AgNPs} /$ RGO nanocomposites to be $I_{\mathrm{D}} / I_{\mathrm{G}}=1.2$, as compared with 1.00 for RGO. The moderate increase in the $I_{\mathrm{D}} / I_{\mathrm{G}}$ intensity ratio indicates the reduction of the exfoliated RGO in the AgNPs/RGO nanocomposites. The crystalline structure of the AgNPs/RGO nanocomposites was analyzed by XRD analysis as displayed in Fig. 2b. The diffraction peaks observed at $38.1^{\circ}, 44.3^{\circ}$ and $64.4^{\circ}$ were consistent with the (111), (200) and (220) planes of Ag crystals. In addition, a broad diffraction peak is detected at $2 \theta=$ $23.5^{\circ}$, representing the (002) plane of the graphitic carbon in the nanocomposites. ${ }^{6}$ These results show that crystalline AgNPs were successfully deposited on the surface of the RGO nanosheets.

\subsection{Electrochemical characterization of AgNPs/RGO/MGCE (RGO : Ag $=1: 0.25$ and $1: 0.75$ )}

The cyclic voltammetry responses of the bare MGCE, and the MGCEs modified with RGO and the different mass ratio AgNPs/ RGO nanocomposites (RGO : $\mathrm{Ag}=1: 0.25$ and $1: 0.75$ ) were examined using the redox couple in $5 \mathrm{mM} \mathrm{K}_{3}\left[\mathrm{Fe}(\mathrm{CN})_{6}\right]$ containing 0.1 M KCl solution (Fig. 3a). Compared with the bare MGCE, the anodic and cathodic peaks for the MGCEs modified with RGO and the AgNPs/RGO nanocomposites were decreased. The anodic and cathodic peaks in the AgNPs/RGO nanocomposite-modified MGCE decrease with increasing Ag content, indicating that the
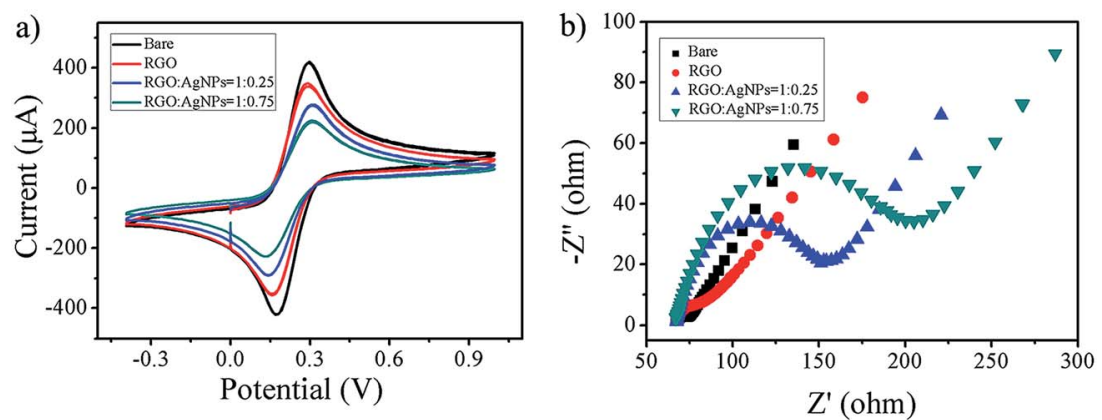

Fig. 3 (a) Cyclic voltammograms and (b) electrochemical impedance spectra obtained on the bare MGCE, and the MGCEs modified with RGO and the different mass ratio AgNPs/RGO nanocomposites in an electrolyte solution of $5 \mathrm{mM} \mathrm{K}_{3}\left[\mathrm{Fe}(\mathrm{CN})_{6}\right]$ and $0.1 \mathrm{M} \mathrm{KCl}^{\text {. Scan rate: } 100 \mathrm{mV} \mathrm{s}}{ }^{-1}$, polarization potential: $0.25 \mathrm{~V}$, frequency spectra: $1-100000 \mathrm{~Hz}$. 

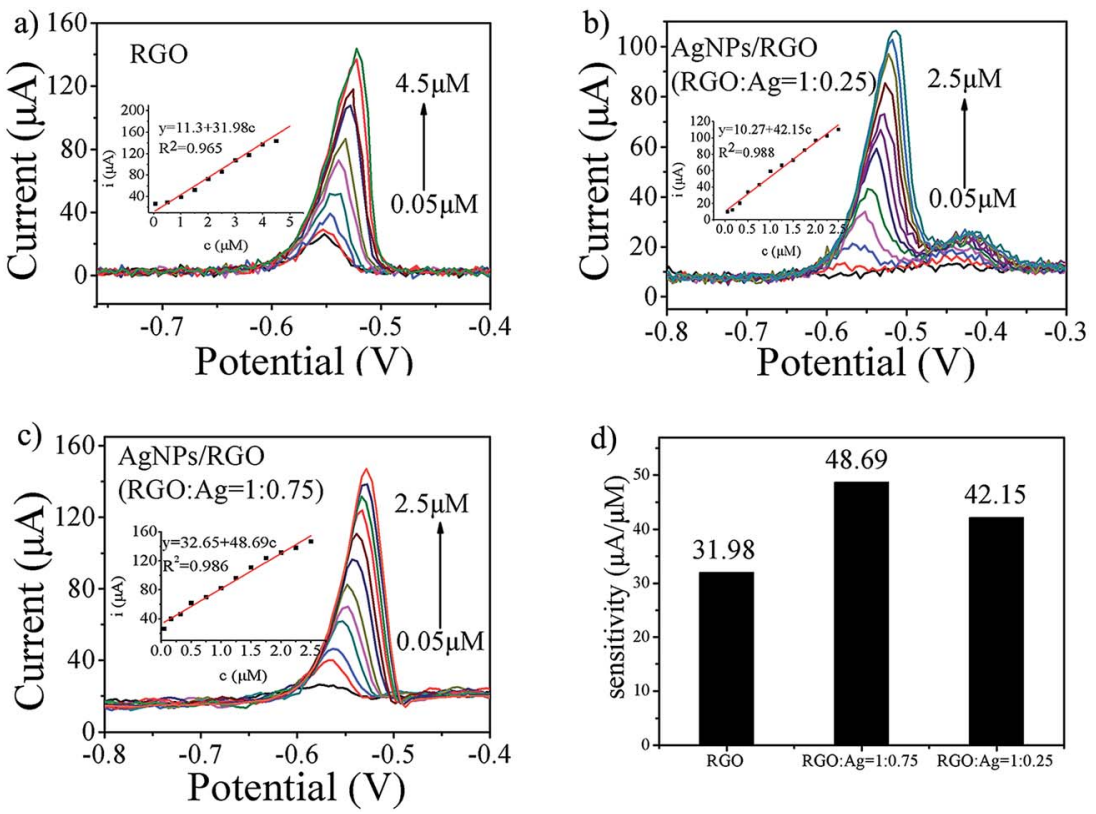

Fig. 4 (a-c) SWASV response toward $\mathrm{Pb}(\Perp)$ detection on $\mathrm{RGO}: \mathrm{Ag}=1: 0.25$ and $1: 0.75$ modified $\mathrm{MGCE}$ electrodes in $0.1 \mathrm{M} \mathrm{HAc}-\mathrm{NaAc}$ (pH $=$ 5.0 ) at the deposition potential of $-1.0 \mathrm{~V}$ for $150 \mathrm{~s}$. The potential step is $5 \mathrm{mV}$, the amplitude is $20 \mathrm{mV}$, and the frequency is $25 \mathrm{~Hz}$. (d) Comparison between the sensitivity versus $\mathrm{Pb}(॥)$ for the modified electrodes based on the different materials considered in this work.

presence of RGO and AgNPs/RGO slows down the electron transfer in the MGCE. On one hand, this phenomenon was attributed to hydroxyl and carboxyl functional groups from $\mathrm{RGO},{ }^{29,30}$ which can hinder the diffusion of ions and the electron transfer to the electrode surface. On the other hand, the AgNPs on RGO are relatively stable and possess weak redox properties.

EIS was used to further characterize the interface properties in the AgNPs/RGO/MGCE samples. In a typical Nyquist plot, the semicircle portion represents the electron-transfer resistance (Ret) in the high-frequency range, while the linear part corresponds to the low-frequency range, where a diffusion-limited process occurs. ${ }^{31}$ As shown in Fig. $3 \mathrm{~b}$, the Ret value of the bare MGCE is very small. The size of the semicircle domain, and therefore the Ret value, was increased by the presence of RGO. The addition of AgNPs further increased the Ret value. This confirms that the presence of the AgNPs/RGO nanocomposite on the electrode surface limits the electron-transfer on the electrochemical probe.

\subsection{Optimization of the modified electrodes}

In order to obtain higher detection sensitivity, modified electrodes were tested under the same conditions. The SWASV responses towards $\mathrm{Pb}$ (II) for the RGO/MGCE and both AgNPs/ RGO/MGCE nanocomposites $(1: 0.25$ and $1: 0.75)$ are displayed in Fig. 4a-c. An obvious peak corresponding to the electrochemical detection of $\mathrm{Pb}$ (II) is clearly observed at about -0.57 V. Furthermore, as seen in Fig. 4 d, significantly different sensitivities towards $\mathrm{Pb}$ (II) detection were associated with each modified electrode material. Interestingly, the $\mathrm{Pb}$ (II) detection sensitivity of the $(1: 0.75)$ AgNPs/RGO/MGCE was the highest. Then follows the $(1: 0.25)$ sample and the RGO-modified electrode. This indicates that the more AgNPs are present, the higher the sensitivity for the detection of heavy metal ions is. The increase in the stripping peak current shows that the reduction of $\mathrm{Pb}(\mathrm{II})$ to $\mathrm{Pb}(0)$ was more efficient at the AgNPs/RGO nanocomposite surface. It is thus clear that $\mathrm{Pb}$ (II) was selectively deposited on top of AgNPs/RGO. The high sensitivity versus $\mathrm{Pb}$ (II) detection may rely on the presence of hydroxyl groups, providing a strongly hydrophilic surface. Table 1 summarizes and compares the sensitivity and detection limit of our modified electrodes with previous studies on $\mathrm{Pb}$ (II) electrochemical sensing. The results demonstrate that a high sensitivity towards $\mathrm{Pb}$ (II) was achieved with the best performing RGO : $\mathrm{Ag}=1: 0.75$ electrode, which was selected for the subsequent detection of multiple heavy metal ions.

Table 1 Comparison of the current sensitivity with previously reported values of different electrodes

\begin{tabular}{|c|c|c|c|}
\hline Electrode & $\begin{array}{l}\text { Sensitivity } \\
\left(\mu \mathrm{A} \mu \mathrm{M}^{-1}\right)\end{array}$ & $\operatorname{LOD}(\mu \mathrm{M})$ & Ref. \\
\hline $\mathrm{RGO} / \mathrm{GCE}$ & 31.98 & 0.533 & This work \\
\hline $\begin{array}{l}\text { AgNPs/RGO/GCE } \\
(\mathrm{RGO}: \mathrm{Ag}=1: 0.25)\end{array}$ & 42.15 & 0.141 & This work \\
\hline $\begin{array}{l}\text { AgNPs/RGO/GCE } \\
(\mathrm{RGO}: \mathrm{Ag}=1: 0.75)\end{array}$ & 48.69 & 0.155 & This work \\
\hline AuNPs/RGO/GCE & 47.76 & $12.69 \mathrm{nM}$ & 26 \\
\hline Band $\mathrm{Fe}_{3} \mathrm{O}_{4} / \mathrm{rGO}$ GCE & 13.6 & 0.169 & 36 \\
\hline $\mathrm{Fe}_{3} \mathrm{O}_{4} / \mathrm{MWCNTS}$ GCE & 11.4 & $6.0 \mathrm{pM}$ & 37 \\
\hline $\mathrm{MnO}_{2} / \mathrm{GCE}$ & 22.4 & - & 38 \\
\hline RGO/MWCNTs GCE & 42.1 & 0.052 & 39 \\
\hline $\mathrm{SnO}_{2} /$ graphene/GCE & 18.6 & 0.0018 & 28 \\
\hline Porous $\mathrm{Co}_{3} \mathrm{O}_{4} / \mathrm{GCE}$ & 71.5 & 0.018 & 40 \\
\hline
\end{tabular}




\subsection{Electrochemical detection of heavy metal ions with AgNPs/RGO/MGCEs}

The detection of heavy metal ions by the AgNPs/RGO $(1: 0.75)$ electrode, including $\mathrm{Pb}(\mathrm{II}), \mathrm{Cd}(\mathrm{II}), \mathrm{Cu}(\mathrm{II})$ and $\mathrm{Hg}$ (II), was performed under similar experimental conditions with individual ions or simultaneously. Fig. 4c shows the SWASV response to $\mathrm{Pb}$ (II) over the metal ion concentration range from 0.05 to 2.5
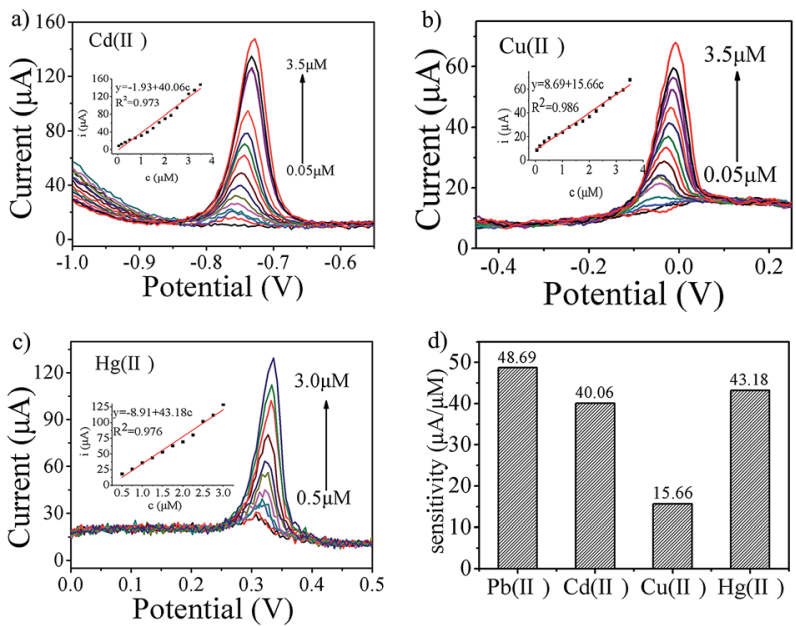

Fig. 5 (a-c) SWASV responses and the corresponding calibration plots of the AgNPs/RGO nanocomposite-modified MGCE for the individual determination of $\mathrm{Cd}\left({ }^{\prime \prime}\right), \mathrm{Cu}(I)$, and $\mathrm{Hg}\left({ }^{\prime \prime}\right)$ after $150 \mathrm{~s}$ in $0.1 \mathrm{M} \mathrm{HAc}-\mathrm{NaAc}$ solution ( $\mathrm{pH}=5.0$ ). Deposition potential: $-1.0 \mathrm{~V}$; amplitude: $25 \mathrm{mV}$; increment potential: $4 \mathrm{mV}$; frequency: $15 \mathrm{~Hz}$. (d) Comparison between the sensitivity versus $\mathrm{Pb}(॥), \mathrm{Cd}(॥), \mathrm{Cu}\left(\|_{)}\right.$and $\mathrm{Hg}(॥)$ of the AgNPs/RGO nanocomposite-modified electrode. $\mu \mathrm{M}$ in $0.1 \mathrm{M}$ HAc-NaAc $(\mathrm{pH}=5.0)$. The $\mathrm{Pb}$ (II) peak is clearly seen at about $-0.56 \mathrm{~V}$, the linearization equation is $i / \mu \mathrm{A}=32.65+$ $48.69 c / \mu \mathrm{M}$, with a correlation coefficient of 0.986 (inset of Fig. 4c), the sensitivity for electrochemical analysis of $\mathrm{Pb}$ (II) is $48.69 \mu \mathrm{A} \mu \mathrm{M}^{-1}$, and the limit of detection (LOD) is calculated to

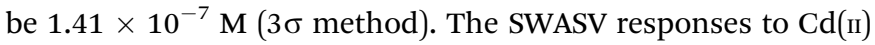
over the concentration range from 0.05 to $3.5 \mu \mathrm{M}$ are shown in Fig. 5a. The Cd(II) peak is visible at about $-0.74 \mathrm{~V}$. The linearization equation is $i / \mu \mathrm{A}=-1.93+40.06 c / \mu \mathrm{M}$, with a correlation coefficient of 0.973 (inset of Fig. 5a) and with a sensitivity of $40.06 \mu \mathrm{A} \mu \mathrm{M}^{-1}$. The limit of detection (LOD) is found to be $2.54 \times 10^{-7} \mathrm{M}$. The SWASV responses to $\mathrm{Cu}(\mathrm{II})$ and $\mathrm{Hg}$ (II) over the respective concentration ranges of 0.05 to $3.5 \mu \mathrm{M}$ and 0.5 to $3.0 \mu \mathrm{M}$ are displayed in Fig. $5 \mathrm{~b}$ and c. Peaks are seen at $-0.05 \mathrm{~V}$ for $\mathrm{Cu}(\mathrm{II})$ and $+0.32 \mathrm{~V}$ for $\mathrm{Hg}$ (II), and the linearization equations for the electrochemical detection are $i / \mu \mathrm{A}=8.69+15.66 \mathrm{c} / \mu \mathrm{M}$ and $i / \mu \mathrm{A}=-8.91+43.18 c / \mu \mathrm{M}$, respectively. The correlation coefficients for $\mathrm{Cu}$ (II) and $\mathrm{Hg}$ (II) are 0.986 and 0.976 (inset of Fig. $5 \mathrm{~b}$ and c), corresponding to a sensitivity of 15.66 and 43.18 $\mu \mathrm{A} \mu \mathrm{M}^{-1}$ and a detection limit of $1.78 \times 10^{-7} \mathrm{M}$ for $\mathrm{Cu}$ (II) and $2.85 \times 10^{-7} \mathrm{M}$ for $\mathrm{Hg}$ (II). The sensitivities are compared in Fig. $5 d$.

As shown in Fig. 6a, different concentrations of heavy metal ions ( $\mathrm{Pb}$ (II), $\mathrm{Cd}$ (II), $\mathrm{Cu}(\mathrm{II})$, and $\mathrm{Hg}$ (II)) were simultaneously detected under similar conditions. The results show that the detection (individual or simultaneous) of the four considered heavy metal ions results in well-separated stripping peaks, respectively observed at $-0.56 \mathrm{~V},-0.74 \mathrm{~V},-0.05 \mathrm{~V}$ and $+0.32 \mathrm{~V}$. It is thus easy to distinguish the four heavy metal ions from the potential of the stripping peak with our modified electrode. Fig. $6 \mathrm{~b}$ shows the interference between the four metal ions. In
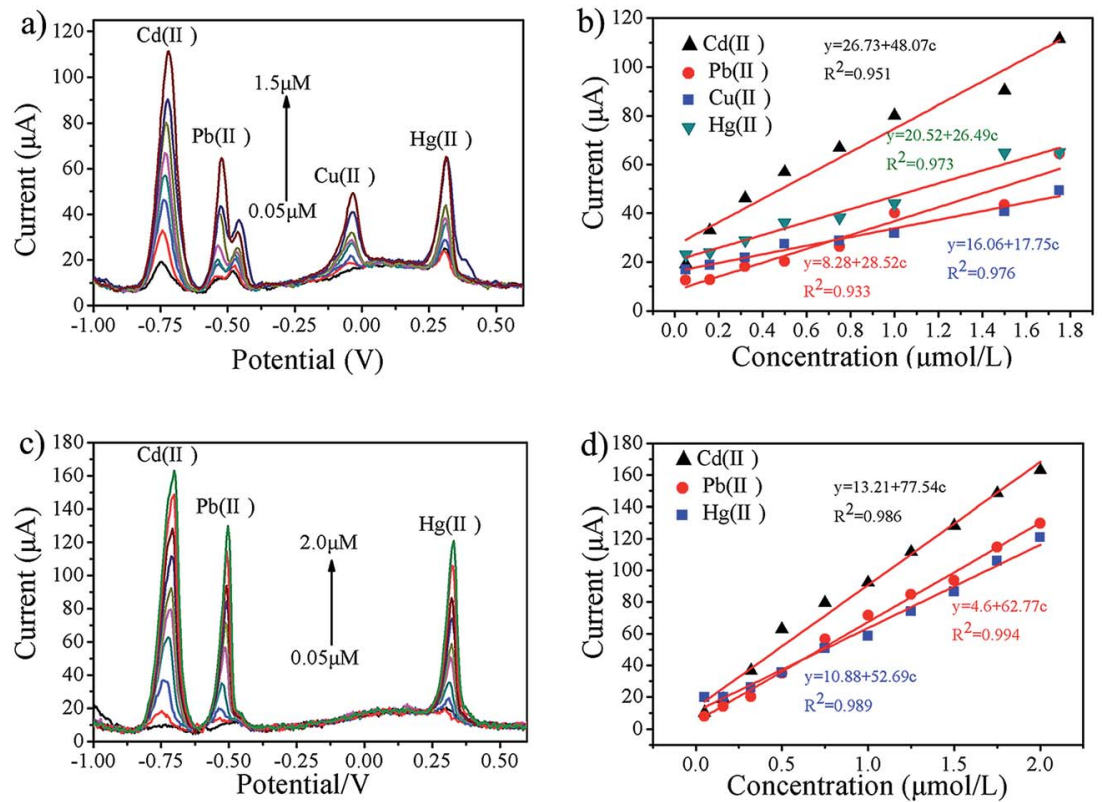

Fig. 6 (a) SWASV responses of the AgNPs/RGO nanocomposite-modified MGCE for the simultaneous detection of $\mathrm{Cd}(\Perp), \mathrm{Pb}(॥), \mathrm{Cu}(॥)$, and $\mathrm{Hg}(॥)$

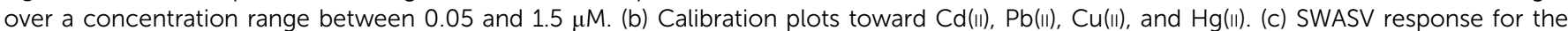
simultaneous detection of $\mathrm{Cd}(॥), \mathrm{Pb}(॥)$, and $\mathrm{Hg}(॥)$ over a concentration range between 0.05 and $2.0 \mu \mathrm{M}$. Deposition potential: $-1.2 \mathrm{~V}$, amplitude: $20 \mathrm{mV}$, frequency: $25 \mathrm{~Hz}$ and vs. $\mathrm{Ag} / \mathrm{AgCl} / \mathrm{KCl}(3 \mathrm{M} \mathrm{KCl}$ saturated with $\mathrm{AgCl})$. (d) Calibration plots toward $\mathrm{Cd}(॥), \mathrm{Pb}(॥)$, and $\mathrm{Hg}(॥)$. 
order to better analyze the interference of these four metal ions, Table 2 displays the sensitivity, correlation coefficient, and calculated LODs for the individual and simultaneous detection of each metal ion. In simultaneous detection, the sensitivity versus $\mathrm{Cu}(\mathrm{II})$ and $\mathrm{Cd}(\mathrm{II})$ is significantly enhanced; this is ascribed to the presence of a $\mathrm{Hg}$ film during the enrichment process. ${ }^{32}$ However, the sensitivity versus $\mathrm{Pb}$ (II) and $\mathrm{Hg}$ (II) is significant decreased, which is attributed to the adsorption of ions from the solution. ${ }^{33}$ In the field of electrochemical detection of heavy metal ions, the hanging mercury drop electrode (HMDE) has been widely used to improve the detection sensitivity. ${ }^{41}$ Fig. $6 \mathrm{a}$ shows that the stripping peak of $\mathrm{Pb}$ (II) is a side-by-side bimodal, with peaks appearing respectively at $-0.56 \mathrm{~V}$ and $-0.46 \mathrm{~V}$. This could be caused by the presence of metal ions on the AgNPs/ RGO electrode surface. ${ }^{34,35}$ This phenomenon implies that the nature of the $\mathrm{Pb}(0)$-enriched AgNPs/RGO nanocomposite electrode surface may have changed. As seen from Fig. 6c, the double stripping peak of $\mathrm{Pb}$ (II) obviously disappears when $\mathrm{Cd}(\mathrm{II})$, $\mathrm{Pb}$ (II) and $\mathrm{Hg}$ (II) coexist; only one single stripping peak for $\mathrm{Pb}$ (II) appears at $-0.56 \mathrm{~V}$. In contrast to Fig. $6 \mathrm{a}$, this phenomenon was associated with the disappearance of the $\mathrm{Cu}$ (II) peak. In addition, when $\mathrm{Pb}$ (II), $\mathrm{Hg}$ (II) and $\mathrm{Cd}$ (II) are detected at the same time (Fig. 6d), the sensitivity versus $\mathrm{Cu}$ (II) and $\mathrm{Cd}(\mathrm{II})$ detection was significantly enhanced; this can be ascribed to the formation of inter-metallic assemblies of the two metals during the enrichment process when both $\mathrm{Cu}^{2+}$ and $\mathrm{Pb}^{2+}$ are present. ${ }^{42}$

\subsection{Stability measurements}

The stability of the AgNPs/RGO nanocomposite-modified electrode is vital in practical applications. The cycling performance of the AgNPs/RGO nanocomposite-modified electrode towards the detection of $\mathrm{Cd}(\mathrm{II})$ at $1.0 \mu \mathrm{M}$ after 5 days of storage in air is displayed in Fig. 7a. It is clear that the stripping currents are nearly constant with cycling and remain steady after 9 cycles. Up to $98.1 \%$ of the peak current was preserved after 9 cycles and 5 days. These results demonstrate that the AgNPs/RGO nanocompositemodified electrode showed good cycling stability and possible long-term durability as a sensor for detecting heavy metal ions. In order to further study the kinetics and cycling stability, EIS was performed on the AgNPs/RGO electrode before and after the 9 cycle test (Fig. 7b). Clearly, there was almost no change in ESR before and after the ageing test, demonstrating that the internal

Table 2 Comparison between individual and simultaneous metal ion detection

\begin{tabular}{lllll}
\hline & & & $\begin{array}{l}\text { Correlation } \\
\text { coefficient }\end{array}$ & $\begin{array}{l}\text { Sensitivity } \\
\left(\mu \mathrm{Amol}^{-1}\right)\end{array}$ \\
\hline Andividual analysis & $\mathrm{Lb}(\mathrm{II})$ & 0.141 & 0.986 & 48.69 \\
& $\mathrm{Cd}(\mathrm{II})$ & 0.254 & 0.973 & 40.06 \\
& $\mathrm{Cu}(\mathrm{II})$ & 0.178 & 0.986 & 15.66 \\
& $\mathrm{Hg}(\mathrm{II})$ & 0.285 & 0.976 & 43.18 \\
Simultaneous analysis & $\mathrm{Pb}(\mathrm{II})$ & 0.287 & 0.933 & 28.52 \\
& $\mathrm{Cd}(\mathrm{II})$ & 0.245 & 0.951 & 48.07 \\
& $\mathrm{Cu}(\mathrm{II})$ & 0.171 & 0.976 & 17.75 \\
& $\mathrm{Hg}(\mathrm{II})$ & 0.180 & 0.973 & 26.49
\end{tabular}
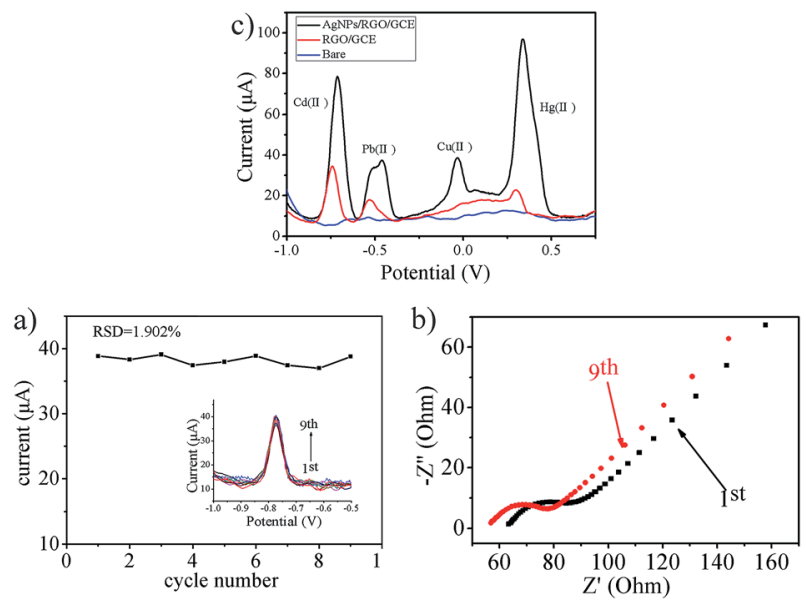

Fig. 7 (a) SWASV responses towards Cd(॥) and (b) EIS of the AgNPs/ RGO nanocomposite electrode in the 1st cycle and after the 9th cycle. (c) SWASV response for the simultaneous detection of $1 \mu \mathrm{M} \mathrm{Cd}(॥), \mathrm{Pb}(॥)$, $\mathrm{Cu}(॥)$ and $\mathrm{Hg}(॥)$ by a bare glassy carbon electrode, RGO/GCE and AgNPs/RGO/GCE.

resistance of the AgNPs/RGO nanocomposite was slightly affected. Nyquist plots also indicate that no significant change in Rct occurs before and after the test. The SWASV response for the simultaneous detection of $1 \mu \mathrm{M} \mathrm{Cd}$ (II), $\mathrm{Pb}$ (II), $\mathrm{Cu}$ (II) and $\mathrm{Hg}$ (II) by a bare glassy carbon electrode, RGO/GCE and AgNPs/RGO/GCE is displayed in Fig. 7c. The results show that there is no obvious stripping peak for the bare glassy carbon electrode, while the stripping peaks of $\mathrm{Cd}$ (II), $\mathrm{Pb}$ (II), $\mathrm{Cu}$ (II) and $\mathrm{Hg}$ (II) by the AgNPs/ $\mathrm{RGO} / \mathrm{GCE}$ are respectively 2.3, 2, 2.7, and 4 times the height of those with the RGO/GCE. Therefore, the AgNPs/RGO-modified electrode is superior for the simultaneous detection of a variety of heavy metal ions. The AgNPs/RGO nanocomposite is thus suitable to be used as an electrochemical sensing material to detect heavy metal ions.

\section{Conclusion}

In this work, RGO and two different mass ratio AgNPs/RGO nanocomposites were designed and prepared by a facile method. The capacity of these three different materials to electrochemically detect toxic $\mathrm{Pb}$ (II) ions was investigated in detail. It was found that the three modified electrodes allow reliable and high sensitivity analysis of $\mathrm{Pb}$ (II) species. The higher the AgNP content, the higher the sensitivity; thus the sensitivity associated with the RGO-modified electrode was the lowest. Furthermore, the best performing AgNPs/RGO (RGO : Ag $=1: 0.75)$ nanocomposite-modified electrode was used as the working electrode for the individual and simultaneous determination of heavy metal ions, such as $\mathrm{Cd}(\mathrm{II}), \mathrm{Pb}(\mathrm{II})$, $\mathrm{Cu}$ (II) and $\mathrm{Hg}$ (II). The electrochemical results demonstrated a high sensitivity, and a low detection limit for the simultaneous detection of $\mathrm{Cd}$ (II), $\mathrm{Pb}$ (II) $\mathrm{Cu}$ (II) and $\mathrm{Hg}$ (II). The experiments also show that the AgNPs/RGO-modified electrode displays good anti-interference properties. The superior stability of the AgNPs/RGO nanocomposite means it has great 
potential as an electrode material for application in the field of electrochemical detection of heavy metal contamination.

\section{Acknowledgements}

The authors are grateful for the support by the National Natural Science Foundation of China (No. 51622507, 61471255, 61474079, 61501316, 51505324), the Doctoral Fund of MOE of China (No. 20131402110013), and the 863 project (2015AA042601).

\section{References}

1 J. G. Wiener, D. P. Krabbenhoft, G. H. Heinz and A. M. Scheuhammer, Handb. Ecotoxicol., 2003, 2, 409-463.

2 I. A. Darwish and D. A. Blake, Anal. Chem., 2002, 74, 52-58.

3 F. B. Edward, C. K. Yap, A. Ismail and S. G. Tan, Water, Air, Soil Pollut., 2009, 196, 297-309.

4 R. Khlifi and A. Hamza-Chaffai, Toxicol. Appl. Pharmacol., 2010, 248, 71-88.

5 S. Yadav, S. Afr. J. Bot., 2010, 76, 167-179.

6 B. Zhang, J. Chen, H. Zhu, T. Yang, M. Zou, M. Zhang and M. Du, Electrochim. Acta, 2016, 196, 422-430.

7 I. Ugulu, Appl. Spectrosc. Rev., 2015, 50, 113-151.

8 L. N. Neupane, E.-T. Oh, H. J. Park and K.-H. Lee, Anal. Chem., 2016, 88, 3333-3340.

9 S.-M. Choi, D.-M. Kim, O.-S. Jung and Y.-B. Shim, Anal. Chim. Acta, 2015, 892, 77-84.

10 M. B. Gumpu, S. Sethuraman, U. M. Krishnan and J. B. B. Rayappan, Sens. Actuators, B, 2015, 213, 515-533.

11 C. Marichy, M. Bechelany and N. Pinna, Adv. Mater., 2012, 24, 1017-1032.

12 L. Matlock-Colangelo and A. J. Baeumner, Lab Chip, 2012, 12, 2612-2620.

13 X. Ren, X. Meng, D. Chen, F. Tang and J. Jiao, Biosens. Bioelectron., 2005, 21, 433-437.

14 H. Im, X.-J. Huang, B. Gu and Y.-K. Choi, Nat. Nanotechnol., 2007, 2, 430-434.

15 J. Lu, I. Do, L. T. Drzal, R. M. Worden and I. Lee, ACS Nano, 2008, 2, 1825-1832.

16 N. J. Ronkainen, H. B. Halsall and W. R. Heineman, Chem. Soc. Rev., 2010, 39, 1747-1763.

17 J. Wang, Chem. Rev., 2008, 108, 814-825.

18 Y. Shao, J. Wang, H. Wu, J. Liu, I. A. Aksay and Y. Lin, Electroanalysis, 2010, 22, 1027-1036.

19 J. Li, S. Guo, Y. Zhai and E. Wang, Electrochem. Commun., 2009, 11, 1085-1088.

20 M. M. Radhi, W. T. Tan, M. Ab Rahman and A. Kassim, Res. J. Appl. Sci., 2010, 5, 59-64.
21 J. Wang, S. B. Hocevar and B. Ogorevc, Electrochem. Commun., 2004, 6, 176-179.

22 Y. Liu, Y. Li and X. P. Yan, Adv. Funct. Mater., 2008, 18, 15361543.

23 X. Nie and W. Hu, Anal. Sci., 2010, 26, 141-142.

24 A. Stein, Z. Wang and M. A. Fierke, Adv. Mater., 2009, 21, 265-293.

25 L.-S. Zhang, W. Li, Z.-M. Cui and W.-G. Song, J. Phys. Chem. C, 2009, 113, 20594-20598.

26 P. Gnanaprakasam, S. E. Jeena, D. Premnath and T. Selvaraju, Electroanalysis, 2016, 28, 1885-1893.

27 R. A. Dar, N. G. Khare, D. P. Cole, S. P. Karna and A. K. Srivastava, RSC Adv., 2014, 4, 14432-14440.

28 Y. Wei, C. Gao, F.-L. Meng, H.-H. Li, L. Wang, J.-H. Liu and X.-J. Huang, J. Phys. Chem. C, 2011, 116, 1034-1041.

29 H. He, J. Klinowski, M. Forster and A. Lerf, Chem. Phys. Lett., 1998, 287, 53-56.

30 A. Lerf, H. He, M. Forster and J. Klinowski, J. Phys. Chem. B, 1998, 102, 4477-4482.

31 Y. Wei, L.-T. Kong, R. Yang, L. Wang, J.-H. Liu and X.-J. Huang, Chem. Commun., 2011, 47, 5340-5342.

32 C. Gao, X.-Y. Yu, R.-X. Xu, J.-H. Liu and X.-J. Huang, ACS Appl. Mater. Interfaces, 2012, 4, 4672-4682.

33 Y.-F. Sun, W.-K. Chen, W.-J. Li, T.-J. Jiang, J.-H. Liu and Z.-G. Liu, J. Electroanal. Chem., 2014, 714, 97-102.

34 L. Wang, W.-H. Xu, R. Yang, T. Zhou, D. Hou, X. Zheng, J.-H. Liu and X.-J. Huang, Anal. Chem., 2013, 85, 3984-3990.

35 L. A. Hutton, M. E. Newton, P. R. Unwin and J. V. Macpherson, Anal. Chem., 2011, 83, 735-745.

36 Y. Sun, W. Zhang, H. Yu, C. Hou, D.-s. Li, Y. Zhang and Y. Liu, J. Alloys Compd., 2015, 638, 182-187.

37 Y. Yang, Y. You, Y. Liu and Z. Yang, Microchim. Acta, 2013, 180, 379-385.

38 Q.-X. Zhang, H. Wen, D. Peng, Q. Fu and X.-J. Huang, J. Electroanal. Chem., 2015, 739, 89-96.

39 J.-T. Zhang, Z.-Y. Jin, W.-C. Li, W. Dong and A.-H. Lu, J. Mater. Chem. A, 2013, 1, 13139-13145.

40 Z.-G. Liu, X. Chen, J.-H. Liu and X.-J. Huang, Electrochem. Commun., 2013, 30, 59-62.

41 M. A. Ferreira and A. A. Barros, Anal. Chim. Acta, 2002, 459, 151-159.

42 D. F. Tibbetts, J. Davis and R. G. Compton, Fresenius' J. Anal. Chem., 2000, 368, 412-414.

43 Y. Zhang, H. Chi, W. Zhang, Y. Sun, Q. Liang, Y. Gu and R. Jing, Nano-Micro Lett., 2014, 6, 80-87. 